\title{
Baseline EEG Pattern on Continuous ICU EEG Monitoring and Incidence of Seizures
}

\author{
Christa B. Swisher, * Dharmen Shah, * Saurabh R. Sinha, *† and Aatif M. Husain*†
}

\begin{abstract}
Purpose: To identify the probability of detecting nonconvulsive seizures based on the initial pattern seen in the first 30 minutes of continuous EEG (cEEG) monitoring.

Methods: Continuous EEG monitoring reports from 243 adult patients were reviewed, assessing the baseline cEEG monitoring pattern and the presence of seizures during the entire monitoring period. The baseline EEG patterns were classified into nine categories: seizures, lateralized periodic discharges, generalized periodic discharges, focal epileptiform discharges, burst suppression, asymmetric background, generalized slowing, generalized periodic discharges with triphasic morphology, and normal.

Results: Overall, 51 patients $(21 \%)$ had nonconvulsive seizures at any time during cEEG monitoring. Notably, 112 patients had generalized slowing as the initial EEG pattern, and none of these patients were noted to have seizures. Seizure rates among the types of baseline EEG findings were as follows: lateralized periodic discharges $(56 \%, \mathrm{n}=9)$, burst suppression $(50 \%, \mathrm{n}=10)$, generalized periodic discharges $(50 \%, \mathrm{n}=2)$, normal $(33 \%$, $\mathrm{n}=3)$, focal epileptiform discharges $(31 \%, \mathrm{n}=35)$, and asymmetric background $(11 \%, \mathrm{n}=46)$.

Conclusions: Patients with only generalized slowing seen on the baseline EEG recording are unlikely to develop seizures on subsequent cEEG monitoring. Depending on the clinical circumstance, the standard duration of cEEG recording (24-48 hours) may be unnecessary in patients with generalized slowing as their only cEEG abnormality.
\end{abstract}

Key Words: Continuous ICU EEG monitoring, Nonconvulsive seizures, Nonconvulsive status epilepticus, EEG.

(J Clin Neurophysiol 2015;32: 147-151)

$\mathrm{N}$ onconvulsive seizures (NCS) and nonconvulsive status epilepticus (NCSE) occur in critically ill patients, and they are seen more frequently in patients with acute or chronic neurologic injury. Previous retrospective and prospective studies have shown the prevalence rate of seizures in neurologic intensive care units to be $8 \%$ to $48 \%$ (Claassen et al., 2004, 2007; DeLorenzo et al., 1998; Jordan, 1995; Oddo et al., 2009; Pandian et al., 2004; Privitera et al., 1994; Towne et al., 2000; Vespa et al., 1999; Vespa et al., 2003; Shafi et al., 2012). Of patients with NCS identified on continuous EEG (cEEG), 14\% to $76 \%$ will have NCSE (DeLorenzo et al., 1998; Jordan, 1995; Pandian et al., 2004; Privitera et al., 1994).

From the *Department of Neurology, Duke University Medical Center, Durham, North Carolina, U.S.A.; and $†$ Neurodiagnostic Center, Veterans Affairs Medical Center, Durham, North Carolina, U.S.A.

Presented as a poster at the American Clinical Neurophysiology Society Meeting, Miami, Florida, U.S.A., February 2013.

Address correspondence and reprint requests to Aatif M. Husain, MD, 299B Hanes House, 330 Trent Drive, Box 102350 Duke University Medical Center, Durham, NC 27710, U.S.A.; e-mail: aatif.husain@dm.duke.edu.

Copyright (C) 2014 by the American Clinical Neurophysiology Society

ISSN: 0736-0258/14/3202-0147
Because routine EEGs detect less than $50 \%$ of seizures that will eventually be noted in critically ill patients (Pandian et al., 2004), a routine EEG is often not sufficient to rule out seizures in patients admitted to the intensive care unit (ICU). Studies have shown that the majority of patients with seizures (88\%) have their first seizure within the first 24 hours of cEEG monitoring, and almost all patients (93\%) have their first seizure occur within 48 hours (Claassen et al., 2004). Based on a survey of epileptologists, most continue cEEG monitoring for 24 hours if no seizures are detected, although a large percentage preferred to monitor for 48 hours, especially if lateralized periodic discharges (LPDs) were seen (Abend et al., 2010). Although cEEG monitoring is necessary to detect NCS and NCSE, it is also very cost and resource intensive.

There is little guidance in the literature regarding ideal duration of cEEG monitoring. One study found that the lack of epileptiform discharges early in cEEG monitoring (within 4 hours) predicted the absence of seizures on subsequent cEEG monitoring (Shafi et al., 2012). The primary goal of our study was to determine if certain EEG patterns detected on the initial 30 minutes of cEEG recordings of adult patients are unlikely to be associated with the development of NCS, therefore justifying quicker termination of cEEG monitoring. Correlating the baseline EEG pattern with subsequent seizure risk could potentially allow for a simplified way to identify patients that may not need extended EEG monitoring.

\section{METHODS}

Permission to complete the study was first obtained from the Duke Institutional Review Board. All adult patients older than 18 years admitted to Duke University Medical Center from January 1 to December 31, 2007, who underwent cEEG monitoring were included in this study. The patients were identified from the Duke EEG database. Continuous EEG was recorded digitally using electrodes applied according to the International 10-20 System. Patients admitted for spell characterization were excluded from the study because these patients represent a separate patient population. In addition, patients were excluded if the duration of cEEG monitoring was less than 24 hours.

We collected basic demographic information, the admission diagnosis, the time when the first seizure occurred and the approximate duration of cEEG monitoring from the EEG reports. The presence of seizures during the entire monitoring period was retrospectively identified by reviewing all EEG reports for each patient. In addition, the EEG pattern present in the first 30 minutes of cEEG monitoring was noted. The baseline EEG patterns were classified into nine categories: seizures, burst suppression, generalized periodic discharges (GPDs), LPDs, focal epileptiform discharges, asymmetric background, GPDs with triphasic morphology, generalized slowing, and normal. In some patients, there were two background abnormalities present. When a nonepileptiform 
abnormality (asymmetric background, generalized slowing, GPDs with triphasic morphology, and normal) and an epileptiform abnormality (burst supression, LPDs, GPDs, focal epileptiform discharges) were both present, the patient was categorized by the epileptiform abnormality. If focal epileptiform discharges and LPDs, GPDs, or burst suppression was present, the patient was categorized by the periodic pattern. If generalized rhythmic delta activity was present, this was included in the generalized slowing group. If lateralized rhythmic delta activity was present, this was included in the asymmetric slowing group. There were no patients with both LPDs and GPDs.

Patients were grouped into 2 categories based on the primary EEG pattern seen on the first 30 minutes of cEEG monitoring. Patients with LPDs, GPDs, burst suppression, and epileptiform discharges were included in the high-risk group. Patients with asymmetric background, GPDs with triphasic morphology, generalized slowing, and a normal pattern were included in the low-risk group. The EEG patterns used to determine the high-risk group and the low-risk group were based on previous work (Claassen et al., 2004). Patients with seizures identified within the first 30 minutes of cEEG monitoring were not included in the analysis because they had already met the primary end point of seizures. The frequency of seizures observed after the initial 30-minute baseline cEEG was compared between the two groups using the $\chi^{2}$ statistic. In the cohort of patients without seizures during the initial 30 minutes of cEEG monitoring, univariate analysis using $\chi^{2}$ testing or Fisher exact test, as appropriate, was conducted to identify significant associations with subsequent seizures on cEEG. A two-sided $P$ value of $<0.05$ was used to determine statistical significance. All analysis was performed using GraphPad Prism (version 5.00 for Windows, GraphPad Software, San Diego, CA). When sampling zeroes occurred, the statistical software added 0.5 to each cell before calculating the odd's ratio or $P$ value to prevent division by zero.

\section{RESULTS}

A total of 243 adult patients underwent cEEG monitoring from January 1 to December 31, 2007. There were 51 (21\%) patients who had NCS or NCSE at any point during cEEG monitoring (during the initial 30-minute recording and/or subsequent cEEG monitoring). Of these patients, seizures occurred within the first 24 hours in 41 patients $(80 \%)$ and within 48 hours in 47 patients $(92 \%)$ while undergoing cEEG monitoring. By 4 days, seizures were seen in $98 \%$ of patients.

The average age for all patients in this study was $56 \pm 18.7$ years old (range, 16-93 years). The majority (88\%) of all patients were located in an ICU setting (neurologic intensive care units primarily and, to a lesser extent, medical and surgical ICUs). The rest of the patients were located in routine or step-down medical or surgical beds. Continuous EEG monitoring was continued for a median duration of 4 days (range, 1-14 days) in patients in whom NCS or NCSE were recorded and for a median duration of 1 day (range, 1-8 days) in patients in whom NCS or NCSE were not recorded.

Of the 51 patients with seizures seen on cEEG monitoring, $74 \%$ had NCS and $26 \%$ with NCSE. Twenty-three of these 51 patients $(45 \%)$ revealed seizures in the initial 30 minutes of cEEG monitoring. These data are shown in Fig. 1. Of these 23 patients, 12 had LPDs as the main abnormality aside from seizures. The other EEG abnormalities seen in these 23 patients include (in order of descending frequency): focal epileptiform discharges (8 patients), asymmetric background (2 patients), and burst suppression (1 patient).

There were 220 patients who did not have seizures identified on the first 30 minutes of cEEG monitoring, and 28 patients $(13 \%)$ later developed NCS or NCSE on cEEG monitoring (Fig. 1). These patients who did not have seizures on the first 30 minutes of EEG monitoring were further analyzed to identify the subsequent seizure rates on additional cEEG monitoring. There were 9 patients whose initial EEG pattern revealed a primary abnormality of LPDs, and 5 $(56 \%)$ of these patients went on to develop seizures. An initial EEG pattern consisting of burst suppression was seen in 10 patients and 5 patients $(50 \%)$ went on to develop seizures. Two patients were noted to have GPDs initially, and $1(50 \%)$ of these patients later developed seizures. The following EEG patterns were also associated with seizures but to a lesser extent than LPDs, GPDs, and burst suppression: normal (1/3 patients, 33\%), focal epileptiform discharges (11/35 patients, $31 \%)$, and asymmetric background (5/46 patients, $11 \%)$. Notably, 112 patients had generalized slowing as the initial EEG pattern, and none of these patients were noted to have seizures. Similarly, no patients who initially had GPDs with triphasic morphology (three patients) developed seizures. Patients with LPDs, burst suppression, and focal epileptiform discharges had a statistically significant higher likelihood of developing seizures when compared with all other baseline cEEG patterns (odds ratio of $10.22,8.13$, and 4.52 , respectively). Patients with diffuse slowing had a statistically significant lower likelihood of developing seizures on subsequent cEEG monitoring (odds ratio of 0.01) when compared with all other baseline cEEG patterns. Because the odds ratio confidence intervals for GPDs, normal, asymmetric background, and GPDs with triphasic morphology included the value of 1 , these were not statistically significant. These data are shown in Table 1.

Patients in the high-risk group (LPDs, GPDs, burst suppression, and focal epileptiform discharges) were more likely to have seizures compared with the low-risk group (generalized slowing, asymmetric background, GPDs with triphasic morphology, and normal), 22/53 patients (41.5\%) and 6/164 patients $(3.7 \%)$, respectively with an odds ratio of 16.9 (95\% confidence interval, 6.4-44.9; $P<0.0001)$.

Figure 2 shows the time to detection of the first seizure for the various EEG patterns. In patients with focal epileptiform discharges $(n=19)$, all seizures were seen by 48 hours. In patients with LPDs $(\mathrm{n}=17)$, all seizures were seen by 72 hours. In contrast, late seizures ( $>72$ hours) were seen in 1 patient with burst suppression and 1 patient with asymmetric background. Of note, there were 15 patients who underwent cEEG monitoring for more than 4 days who never had seizures detected. Of these 15 patients, 5 had generalized slowing only. The others had LPDs (2), GPDs (1), burst suppression (1), focal epileptiform discharges (2), asymmetric background (3), and GPDs with triphasic morphology (1) in addition to generalized slowing.

The patient population with the highest incidence of seizures identified on cEEG was patients with subdural hematomas $(6 / 14$ patients, $43 \%$ ). This was followed by patients with a central nervous system infection (3/10 patients, 30\%), hypoxic ischemic encephalopathy ( $7 / 24$ patients, $29 \%$ ), patients postoperative from a neurosurgical procedure $(6 / 21$ patients, $29 \%)$, patients with brain tumor (9/34 patients, 26\%), patients admitted with the diagnosis of epilepsy and fluctuating mental status (4/16 patients, 25\%), ischemic stroke $(3 / 17$ patients, $18 \%)$, traumatic brain injury $(4 / 25$ patients, $16 \%)$, subarachnoid hemorrhage $(3 / 20$ patients, $15 \%)$, and toxic/metabolic conditions (3/31 patients, 10\%). Seizures were not 


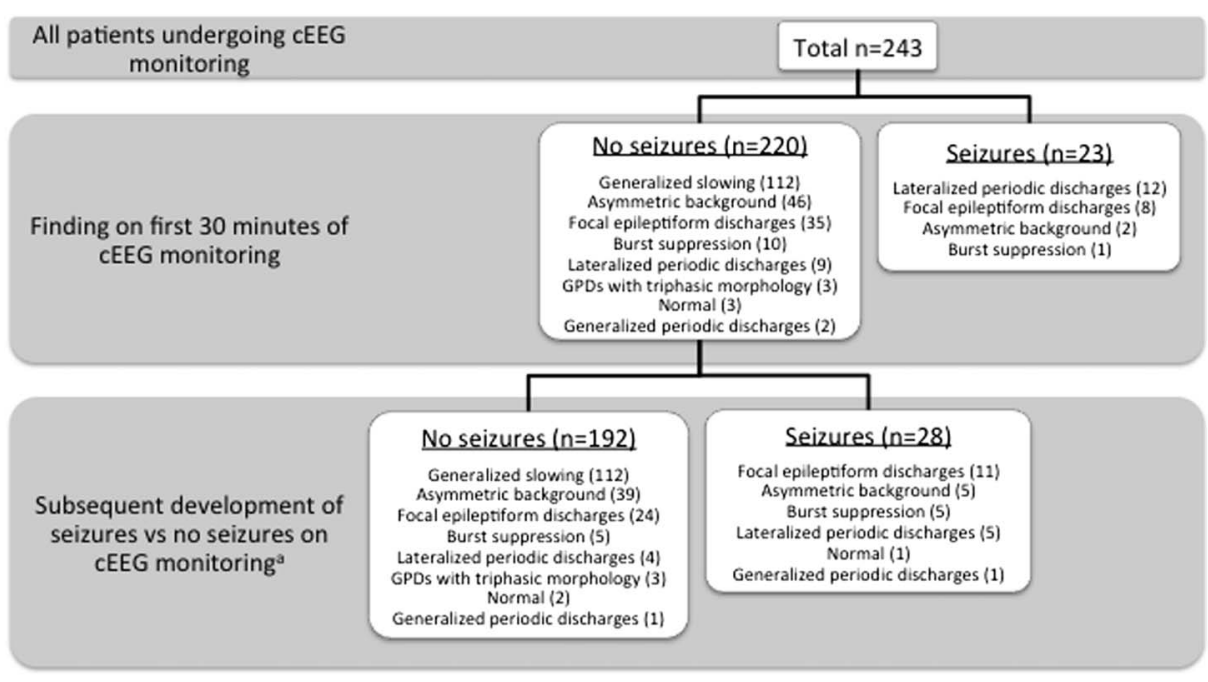

FIG. 1. Overall cEEG results: Total number of patients undergoing $\mathrm{CEEG}$ and the number of patients with and without seizures on the first

30 minutes of $C E E G$ monitoring and subsequent cEEG monitoring results. The specific EEG findings are detailed for each group, and the number of patients is shown within parentheses. apatients were categorized by initial 30-minute baseline EEG pattern. seen in patients admitted with intracerebral hemorrhage (19 patients). Statistical analysis (two-sided Fisher exact test) confirmed that seizures in patients with intracerebral hemorrhage occurred less frequently when compared with seizures in patients with all other diagnoses $(P=0.02)$. These data are shown in Table 2. No single etiology had a significant positive association with the later development of seizures.

\section{DISCUSSION}

In this retrospective study, the overall seizure rate in 243 adult patients who underwent cEEG monitoring was $21 \%$. The seizure rate in our study is similar to previously published seizure rates of $8 \%$ to $35 \%$ studying all patients undergoing cEEG monitoring in a neuroICU, rather than a select patient population (Claassen et al., 2004; Jordan, 1995; Towne et al., 2000). Higher seizure rates (48\%-68\%) were seen in studies that selectively enrolled patients who had a very high risk of having NCS or NCSE, such as patients with convulsive status epilepticus before initiation of cEEG (DeLorenzo et al., 1998; Pandian et al., 2004).

Previous studies have not identified the probability of detecting NCS or NCSE based on the initial EEG pattern seen on cEEG monitoring. Patients in the low-risk group (generalized slowing, GPDs with triphasic morphology, asymmetric background, and normal) had a statistically significant lower risk of seizures. Of the 112 patients in this study with generalized slowing and on their initial cEEG recording, none went on to develop seizures. When compared with all other baseline cEEG patterns, patients with generalized slowing had an odds ratio of 0.01 (relative risk 0.013 ) to develop seizures. These data suggest that it may not be necessary to perform cEEG monitoring for 24 to 48 hours on patients who have generalized slowing as the only abnormality on the initial 30 minutes of cEEG recording. A recent study by Shafi et al. (2012) found similar results in that the lack of epileptiform discharges early in cEEG monitoring (within 4 hours) predicted the absence of seizures on subsequent cEEG monitoring. With the data we present, providers can make an early determination of a patient's seizure risk based on their baseline EEG pattern, rather than waiting to determine when/if the first epileptiform discharge will appear. This may help conserve vital resources that are needed to perform cEEG monitoring.

Patients in the high-risk group (LPDs, GPDs, burst suppression, and focal epileptiform discharges on the initial 30 minutes of cEEG monitoring) had a high association with seizures later in the monitoring (56\%, 50\%, 50\%, and 31\%, respectively). Long-term cEEG monitoring would be appropriate if any of these patterns are noted in the initial 30 minutes of recording. This finding is similar to previous studies by Claassen et al. who found that LPDs and GPDs were seen more frequently among patients with seizures seen on cEEG when compared with patients without seizures (Claassen et al., 2004, 2007).

TABLE 1. Rate of Electrographic Seizures as a Function of Initial Continuous EEG Pattern

\begin{tabular}{lccc}
\hline Initial EEG Recording & Seizure Present, $(\mathbf{N}=\mathbf{2 8}), \mathbf{n}(\mathbf{\%})$ & Seizure Absent, $(\mathbf{N}=\mathbf{1 9 2}), \mathbf{n}(\mathbf{\%})$ & Odds Ratio (95\% CI) \\
\hline LPDs & $5(18)$ & $4(2)$ & $\mathbf{1 0 . 2 2}(\mathbf{2 . 5 6 - 4 0 . 7 9 )}$ \\
GPDs & $1(4)$ & $1(0.5)$ & $7.07(0.43-116.45)$ \\
Burst suppression & $5(18)$ & $5(3)$ & $\mathbf{8 . 1 3}(\mathbf{2 . 1 9}-\mathbf{3 0 . 2 3})$ \\
Normal & $1(4)$ & $2(1)$ & $3.52(0.31-40.13)$ \\
Epileptiform discharges & $11(39)$ & $24(13)$ & $\mathbf{4 . 5 2}(\mathbf{1 . 9 0}-\mathbf{1 0 . 8 2})$ \\
Asymmectric background & $5(18)$ & $41(21)$ & $0.80(0.29-2.24)$ \\
GPDs with triphasic morphology & $0(0)$ & $3(2)$ & $0.95(0.05-18.88)$ \\
Generalized slowing & $0(0)$ & $112(58)$ & $\mathbf{0 . 0 1}(\mathbf{0 . 0 1}-\mathbf{0 . 2 1})$ \\
\hline
\end{tabular}

Bolded values represent statistical significance. The cohort consists of patients without seizures during baseline cEEG recording. Patients with seizures $(\mathrm{n}=23)$ during the 30 minutes of baseline cEEG acquisition are not included.

CI, confidence interval; GPDs, generalized periodic discharges; LPDs, lateralized periodic discharges. 


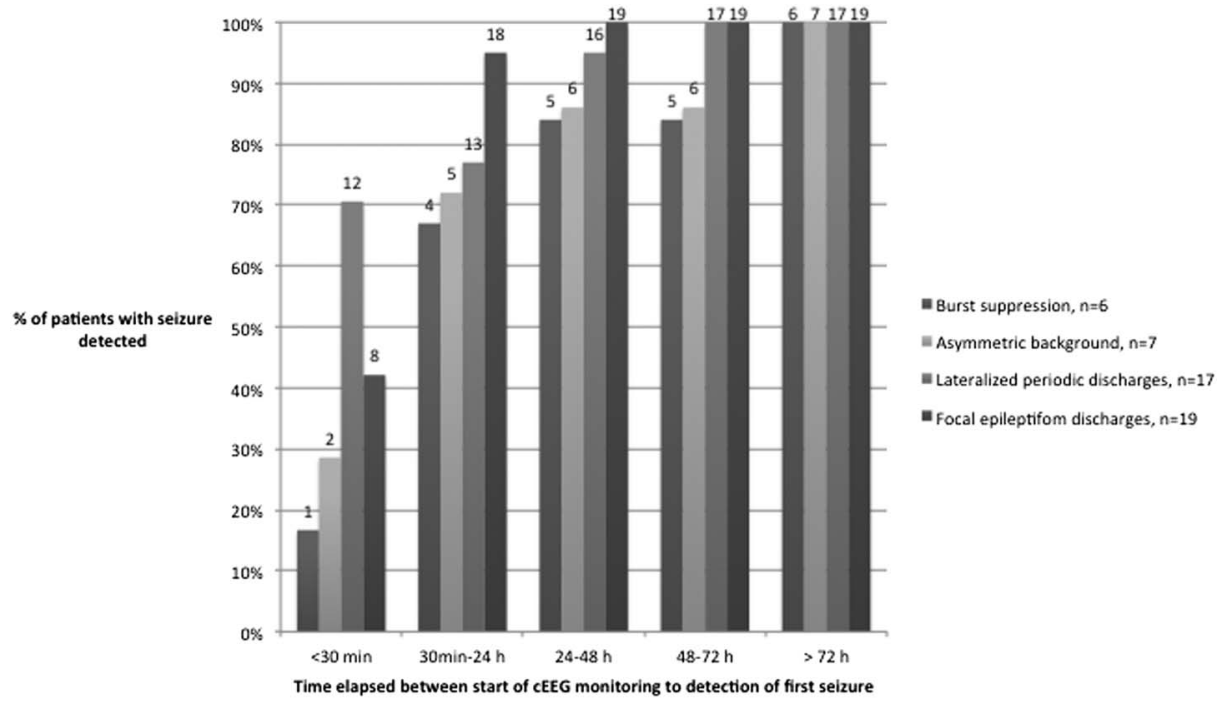

FIG. 2. Time elapsed between start of cEEG monitoring and detection of the first seizure as a function of the initial 30-minute cEEG finding; Only patients who had seizures on cEEG monitoring were included. Patients with generalized slowing and GPDs with triphasic morphology not included as seizures were not detected in these groups. There was 1 patient with GPDs and 1 patient with a normal EEG who each had seizures detected at the 30 minutes to 24 hours time point. These patients were not included because of the low sample size. Patients with seizures on the baseline EEG acquisition were not included in the chart because they already met the primary end point of seizures. Patients were monitored on cEEG for various durations (median, 4 days; range, 1-14 days; interquartile range, 2 days). The total number of patients is shown at the end of each bar.

Additionally, if the EEG background changes from generalized slowing to one containing epileptiform discharges, a longer duration of cEEG monitoring would likely be indicated. Interestingly, a recent case-control study revealed that although GPDs were strongly associated with NCS and NCSE, the presence of GPDs were not independently associated with a worse outcome when compared with controls who were matched for age, etiology, and level of consciousness (Foreman et al., 2012). Although the new ACNS terminology guidelines place triphasic waves as a subcategory of GPDs, we chose to differentiate these two groups because patients with triphasic waves have been found to have a low incidence of NCS (4\%) (Shafi et al., 2012), despite the fact that it is often difficult to discriminate between triphasic waves and NCSE (Kaplan, 2007).

TABLE 2. Subsequent Seizure Rate After Baseline EEG Acquisition as a Function of Admission Diagnosis

\begin{tabular}{lccc}
\hline Diagnosis & $\begin{array}{c}\text { Seizures Present, } \\
(\mathbf{N}=\mathbf{5 1}), \mathbf{n}(\mathbf{\%})\end{array}$ & $\begin{array}{c}\text { Seizures Absent, } \\
(\mathbf{N}=\mathbf{1 9 2}), \mathbf{n}(\mathbf{\%})\end{array}$ & $\boldsymbol{P}$ \\
\hline SDH & $6(12)$ & $8(4)$ & 0.39 \\
CNS infection & $3(6)$ & $7(4)$ & 0.74 \\
HIE & $7(14)$ & $17(9)$ & 0.81 \\
Postop & $6(12)$ & $15(8)$ & 0.86 \\
Brain tumor & $9(18)$ & $25(13)$ & 1.00 \\
Epilepsy related & $4(8)$ & $12(6)$ & 1.00 \\
Other & $3(6)$ & $9(5)$ & 1.00 \\
Ischemic stroke & $3(6)$ & $14(7)$ & 0.77 \\
TBI & $4(8)$ & $21(11)$ & 0.47 \\
SAH & $3(6)$ & $17(9)$ & 0.42 \\
Toxic/metabolic & $3(6)$ & $17(9)$ & 0.07 \\
ICH & $0(0)$ & $19(10)$ & 0.02 \\
\hline
\end{tabular}

Data are given as n (\%) and associated two-tailed $P$ value as calculated by Fisher exact test. Cohort consists of all patients with seizures on baseline EEG recording and/or seizures on subsequent cEEG recording.

CNS, central nervous system; HIE, hypoxic ischemic encephalopathy; ICH, intracerebral hemorrhage; Postop, patients postoperative from a neurosurgical procedure; SAH, subarachnoid hemorrhage; $\mathrm{SDH}$, subdural hematoma; TBI, traumatic brain injury.
EEGs of patients with triphasic waves should always be interpreted with caution for this reason. A trial of an antiepileptic medication is often necessary to make this determination, and various EEG features, such as time-lag, frequency, duration of each phase, the presence of multispikes, and associated background frequency, can be helpful (Kaplan, 2007).

There was one patient with a normal EEG on the baseline recording who subsequently developed seizures on cEEG. This patient was admitted with severe hyponatremia secondary to medication side effect. It is likely that this case represents an outlier as it is typically unusual for a patient with a normal EEG to develop seizures on subsequent cEEG monitoring. Furthermore, it is very unlikely that the seizure rate of $33 \%$ in this study represents the actual seizure rate in patients with a normal background on cEEG monitoring. Patients with a normal EEG often undergo cEEG monitoring $<24$ hours. This study did not include patients with $<24$ hours of cEEG monitoring. If these patients had been included in the study, it is very likely that the seizure rate for patients with a normal EEG would be much lower.

Our study found that the majority of patients with seizures (92\%) had their first seizure within the first 48 hours of monitoring, which is similar to the study by Claassen et al. who found that $98 \%$ of patients without coma and $87 \%$ patients with coma with seizures had the first seizure within 48 hours of monitoring (Claassen et al., 2004). It should be noted, however, that monitoring is rarely continued beyond 48 hours if seizures are not noted (Abend et al., 2010). Because of this and because every ICU patient does not undergo cEEG monitoring, the true incidence of seizures in these critically ill patients is difficult to determine.

Seizure frequencies in certain patient populations in our study were similar to previous reports: central nervous system infection (30\% vs. 25\%-33\% in the literature) (Claassen et al., 2004; Jordan, 1995; Shafi et al., 2012), postoperative from a neurosurgical procedure (29\% vs. 18\%-23\%) (Claassen et al., 2004; Shafi et al., 2012), hypoxic ischemic encephalopathy (29\% vs. 20\%-54\%) (Claassen et al., 2004; DeLorenzo et al., 1998; Shafi et al., 2012), patients with brain tumor $(27 \%$ vs. $17 \%-54 \%)$ (Claassen et al., 2004; DeLorenzo et al., 1998; Jordan, 1995; Shafi et al., 2012), epilepsy-related $(25 \%$ vs. $24 \%-56 \%)$ (Claassen et al., 2004; 
DeLorenzo et al., 1998; Jordan, 1995), ischemic stroke (18\% vs. 6\%-35\%) (Claassen et al., 2004; DeLorenzo et al., 1998; Jordan, 1995; Shafi et al., 2012; Vespa et al., 2003), traumatic brain injury (16\% vs. 8\%-28\%) (Claassen et al., 2004; Jordan, 1995; Shafi et al., 2012; Vespa et al., 1999), and subarachnoid hemorrhage (15\% vs. $13 \%-19 \%$ ) (Claassen et al., 2004; Shafi et al., 2012). The rate of seizures in patients with toxic/metabolic conditions in our study was lower than published reports $(10 \%$ vs. $21 \%-60 \%$ in the literature) (Claassen et al., 2004; DeLorenzo et al., 1998; Jordan, 1995; Shafi et al., 2012). We did not detect seizures in patients with intracerebral hemorrhage in our patient population despite other reports noting relatively high seizure rates in this patient population $(13 \%-28 \%$ reported in the literature) (Claassen et al., 2004, 2007; Vespa et al., 2003). The rate of seizures in patients with subdural hematomas was particularly high (43\%) in this study. The seizure rate in the acute period in patients with subdural hematomas and no other intracranial injury or hemorrhage has not been previously reported.

These results are limited by the relatively small sample size, particularly for each etiology, and retrospective design. Specifically, there were especially small numbers for certain patient groups, such as central nervous system infection, subdural hematomas, and ischemic strokes, as noted above. In addition, there were relatively small numbers for certain EEG patterns, especially GPDs. Despite these limitations, many findings of this study are consistent with other studies in similar patient populations.

Despite the limitations of this study, the results show that seizure rates are high in patients with GPDs, LPDs, burst suppression, and focal epileptiform discharges, and long-term cEEG monitoring is warranted in these patients. Conversely, none of the 112 patients with generalized slowing and GPDs with triphasic morphology on the first 30 minutes of cEEG recording went on to develop seizures on cEEG. Because most providers choose to monitor patients for 24 to 48 hours to rule out seizures, these data suggest that the standard duration of monitoring may not be necessary for all patients with generalized slowing in the initial part of the study. As outliers may occur, sound clinical judgment in conjunction with these results can provide guidance on how long cEEG monitoring should be continued. A larger prospective study is warranted to determine the prognostic value of cEEG baseline recordings.

\section{REFERENCES}

Abend NS, Dlugos DJ, Hahn CD, et al. Use of EEG monitoring and management of non-convulsive seizures in critically ill patients: a survey of neurologists. Neurocrit Care 2010;12:382-389.

Claassen J, Mayer SA, Kowalski RG, et al. Detection of electrographic seizures with continuous EEG monitoring in critically ill patients. Neurology 2004;62:1743-1748

Claassen J, Jette N, Chum F, et al. Electrographic seizures and periodic discharges after intracerebral hemorrhage. Neurology 2007;69:1356-1365.

DeLorenzo RJ, Waterhouse EJ, Towne AR, et al. Persistent nonconvulsive status epilepticus after the control of convulsive status epilepticus. Epilepsia 1998;39:833-840.

Foreman B, Claassen J, Abou Khaled K, et al. Generalized periodic discharges in the critically ill: a case-control study of 200 patients. Neurology 2012;79:1951-1960

Jordan KG. Neuropysiologic monitoring in the neuroscience intensive-care unit Neurol Clin 1995;13:579-626.

Kaplan PW. EEG criteria for nonconvulsive status epilepticus. Epilepsia 2007:48:39-41.

Oddo M, Carrera E, Claassen J, et al. Continuous electroencephalography in the medical intensive care unit. Crit Care Med 2009;37:2051-2056.

Pandian JD, Cascino GD, So EL, et al. Digital video-electroencephalographic monitoring in the neurological-neurosurgical intensive care unit: clinical features and outcome. Arch Neurol 2004;61:1090-1094.

Privitera M, Hoffman M, Moore JL, Jester D. EEG detection of nontonic-clonic status epilepticus in patients with altered consciousness. Epilepsy Res 1994;18:155-166.

Shafi MM, Westover MB, Cole AJ, et al. Absence of early epileptiform abnormalities predicts lack of seizures on continuous EEG. Neurology 2012;79:1796-1801.

Towne AR, Waterhouse EJ, Garnett L. Prevalence of nonconvulsive status epilepticus in comatose patients. Neurology 2000;55:1422-1423.

Vespa PM, Nuwer MR, Nenov V, et al. Increased incidence and impact of nonconvulsive and convulsive seizures after traumatic brain injury as detected by continuous electroencephalographic monitoring. J Neurosurg 1999;91:750-760.

Vespa PM, O'Phelan K, Shah M, et al. Acute seizures after intracerebral hemorrhage: a factor in progressive midline shift and outcome. Neurology 2003;60:1441-1446. 